\title{
Successful Removal of a Left Main Coronary Artery Thrombus Induced by Vasospasm of the Aorta After the Injection of Contrast Medium
}

\author{
Toshihiko Yamasa, MD; Shiro Hata, MD; Atsuki Fukae, MD; \\ Akiyo Ninomiya, MD; Satoshi Ikeda, MD*; \\ Yoshiyuki Miyahara, MD*; Shigeru Kohno, MD*
}

\begin{abstract}
A 51-year-old woman with acute myocardial infarction underwent emergency coronary angiography. The patient had an episode of idiopathic thrombocytopenic purpura, and underwent splenectomy at the age of 36. On admission, platelet count in the peripheral blood was $43.2 \times 10^{4} \mu 1$. During the initial emergency left coronary angiography, a thrombus was detected in the left main trunk. The thrombus was drawn back to the orifice of the left coronary artery, and finally disappeared into the aorta by injecting contrast media. After the thrombus disappeared, no stenotic lesion was detected in the left coronary artery. One month later, when acetylcholine-provocation coronary angiography was performed, marked vasospasm was detected in the left coronary arteries. Coronary thrombosis in the patient might have been induced by a coronary spasm, and the presence of thrombocytosis might also have affected the development of a coronary thrombus. However, it was spontaneously drawn back to the aorta by back flow of contrast media, which was injected via the entrance of the left coronary artery. A case of acute myocardial infarction whose thrombus occluded the left main coronary artery and was removed at first injection of contrast media is presented. (Jpn Circ J 2001; 65: 579-580)
\end{abstract}

Key Words: Coronary vasospasm; Idiopathic thrombocytopenic purpura; Left main coronary artery; Splenectomy; Thrombus

A 51-year-old woman was admitted to hospital by ambulance because of sudden intense chest pain, weakness and dyspnea. Cold sweating was observed on admission. Her pulse rate was 100 beats/min and systolic blood pressure was $68 \mathrm{mmHg}$. Moist rales were audible from the bilateral lung, suggesting a state of shock and heart failure. Electrocardiograms (ECG) demonstrated the presence of a sinus rhythm, complete right bundle branch block, elevated ST segments on I, a $V_{L}$ and $V_{2}$ to $V_{5}$ leads, and lowered ST segment on II, III and aVF leads (Fig 1). Based on these findings, the diagnosis of acute myocardial infarction and cardiogenic shock was established.

The patient had an episode of idiopathic thrombocytopenic purpura, and underwent a splenectomy at the age of 36 years. On admission, platelet count in the peripheral blood was $43.2 \times 10^{4} \mu$ l. Other laboratory findings were all normal except for an increased white blood cell count of $16,300 \mu 1$. Dehydration, which can cause thrombosis, was not observed. Furthermore, levels of both IgG and IgM class anti-cardiolipin antibodies, which frequently cause thrombotic complications, were in the normal range, and lupus anticoagulants were not detected.

During the initial emergency left coronary angiography, a thrombus was detected in the left main trunk. The thrombus was drawn back to the orifice of the left coronary artery,

(Received September 25, 2000; revised manuscript received December 20, 2000; accepted January 10, 2001)

Department of Internal Medicine, Sasebo Municipal General Hospital and *Second Department of Internal Medicine, Nagasaki University School of Medicine, Nagasaki, Japan

Mailing address: Toshihiko Yamasa, MD, Department of Internal Medicine, Sasebo Municipal General Hospital, 9-3 Hirase-machi, Sasebo 857-8511, Japan and finally disappeared into the aorta at first injection of contrast media (Fig 2). After the thrombus disappeared, no stenotic lesion was detected in the left coronary artery. No symptoms nor signs of an embolism caused by the thrombus were observed. Although symptoms, hemodynamics, and ECG findings did not improve after the removal of the thrombus, creatine kinase levels increased to $7,944 \mathrm{IU} / \mathrm{L}$
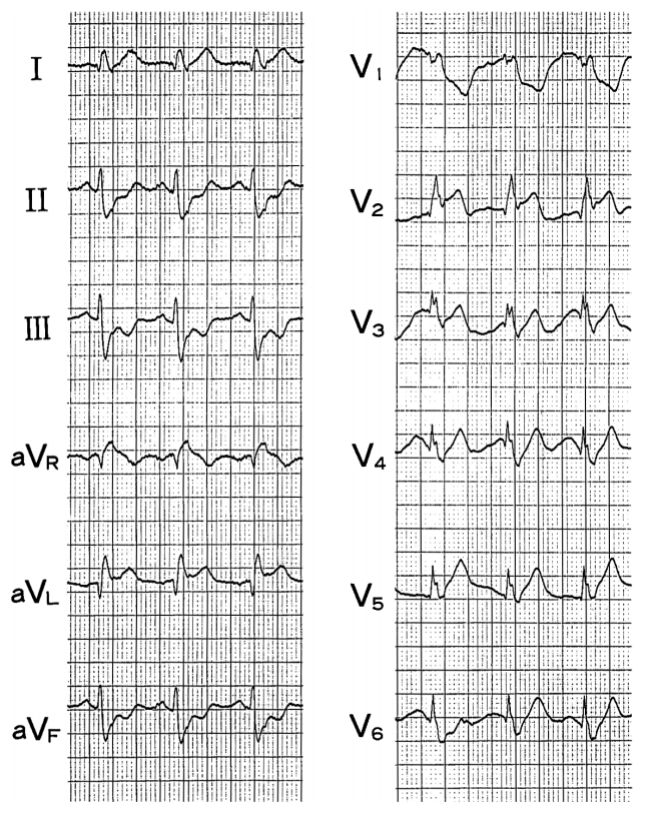

Fig 1. Electrocardiogram on admission. Right bundle branch block and ST segment elevation in the anterior leads were found. 


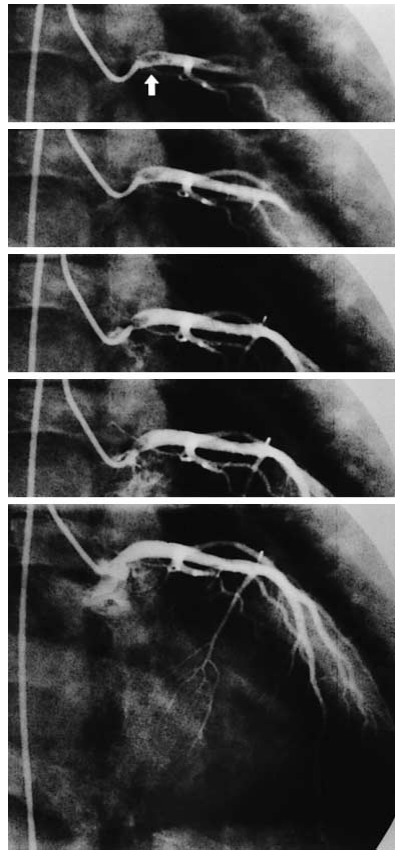

Fig 2. A thrombus (arrow) in the left main trunk. The thrombus was gradually drawn back to the orifice of left coronary artery, and then disappeared into the aorta.

thereafter. Therefore, it was considered that the thrombus was the major cause of myocardial infarction. Although intra-aortic balloon pumping was required to treat heart failure and cardiogenic shock, the symptoms gradually disappeared.

One month later, when acetylcholine-provocation coronary angiography was performed, marked vasospasm was induced in the bilateral coronary arteries, especially in the left anterior descending artery (Fig 3). Simultaneous intravascular ultrasound imaging (IVUS) did not demonstrate any atheroma in the left coronary artery.

\section{Discussion}

It has been reported that coronary spasm rarely causes acute myocardial infarction!, ${ }^{1,2}$ But, in some cases, it can be a cause of myocardial infarction? In an animal experiment, however, Nagasawa and colleagues demonstrated that prolonged coronary spasm induces intramural bleeding in the coronary artery4 Therefore, we speculated that coronary spasm may progress to coronary occlusion. Because marked spasm was induced in the coronary artery of the patient after the administration of acetylcholine, the diagnosis of vasospastic angina was established; however, simultaneous IVUS did not demonstrate any atheroma in the left coronary artery. Hence, it was considered that the coronary thrombosis was not caused by a ruptured atheroma but was partly caused by a coronary spasm. Furthermore, the patient had past histories of idiopathic thrombocytopenic purpura and splenectomy. Therefore, it was concluded that the patient had thrombocytosis.

Nielsen reported a case of acute myocardial infarction that developed after a splenectomy5 However, cases of thrombocytosis are rarely complicated by acute myocardial infarction. It is also reported that patients with primary thrombocytosis exhibiting peripheral platelet counts above $1 \times 10^{5} \mu 1$ are frequently complicated by peripheral arterial
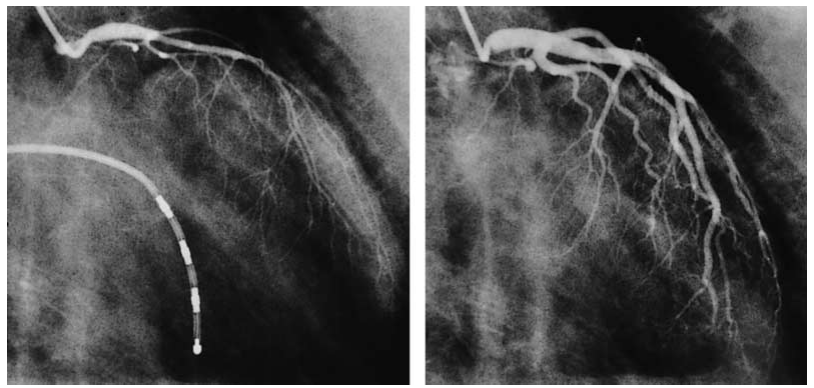

Fig 3. Left anterior oblique view. A acetylcholine provocation coronary angiography by intracoronary injection of acetylcholine. Severe diffuse spasm was induced at the left anterior descending artery and circumflex artery. Total obstruction was observed at the distal portion of left anterior descending artery (left). Coronary spasm was relieved after intracoronary injection of isosorbide dinitrate (right).

thrombosis in the extremities, but rarely complicated by myocardial infarction? Hence, it was considered that both a coronary spasm and thrombocytosis caused the coronary thrombosis in the patient.

Coronary thrombosis in patients with acute myocardial infarction is generally treated by thrombolytic agents or by thrombectomy after destroying the thrombi by balloon angioplasty. Intracoronary thrombolysis using specially designed catheters; that is, ultrasound thrombolysis ${ }^{7}$ or AngioJet, ${ }^{8}$ were reported recently.

Because residual thrombi generally migrate to the distal region of the coronary artery, this is a rare case, in which the coronary thrombi migrated to the proximal region of the coronary artery. To our knowledge, there have been no reports describing similar findings. However, it was fortunate that the thrombi in the patient became movable when the coronary spasm was relieved by the administration of nitrites, and when thrombosis occurring in the major branch of the left coronary artery near its entrance was easily influenced by the back flow of injected contrast media. Because the catheter tip inserted into the left coronary artery was located below the thrombus, the contrast medium was injected to the distal side of the thrombus. Therefore, visualization of the thrombus was influenced by the back flow of the contrast medium.

\section{References}

1. Massseri A, L'Abbate A, Baroldi G, Chierchia S, Marzilli M, Ballestra $\mathrm{AM}$, et al: Coronary vasospasm as a possible cause of myocardial infarction: A conclusion derived from the study of 'preinfarction' angina. N Engl J Med 1978; 299: 1271-1277

2. Nakamura M, Takeshita A, Nose Y: Clinical characteristics associated with myocardial infarction, arrhythmias, and sudden death in patients with vasospastic angina. Circulation 1987; 75: 1110-1116

3. Araki H, Hayata N, Nakamura M: Clinical evidence suggesting vasospastic cause of myocardial infarction. Jpn Circ J 1986; 50: 174-180

4. Nagasawa K, Tomoike H, Hayashi Y, Yamada A, Yamamoto T, Nakamura M: Intramural hemorrhage and endothelial changes in atherosclerotic coronary artery after repetitive episodes of spasm in Xray-irradiated hypercholesterolemic pigs. Circ Res 1989; 65: 272-282

5. Nielsen H: Letalt myokardieinfarkt hos patient med Pstsplenektomitrombocytose. Ugeskr Laeger 1988; 150: 2125-2126 (in German)

6. Singh AK, Wetherley-Mein G: Microvascular occlusive lesion in primary thrombocythaemia. Br J Haematol 1977; 36: 553-564

7. Brosh D, Rosenschein U: Catheter-based ultrasound thrombolysis: A new promising thrombus-debulking device for the treatment of intracoronary thrombosis. Semin Interv Cardiol 2000; 5: 149-155

8. Whisenant BK, Baim DS, Kuntz RE, Garcia LA, Ramee SR, Carrozza JP: Rheolytic thrombectomy with the Possis AngioJet: Technical consideration and initial clinical experience. J Invasive Cardiol 1999; 11: $421-426$ 